\title{
WEAK LOCAL LINEAR DISCRETIZATIONS FOR STOCHASTIC DIFFERENTIAL EQUATIONS WITH JUMPS
}

\author{
F. CARBONELL* AND \\ J. C. JIMENEZ, ${ }^{* *}$ Instituto de Cibernética
}

\begin{abstract}
Weak local linear approximations have played a prominent role in the construction of effective inference methods and numerical integrators for stochastic differential equations. In this note two weak local linear approximations for stochastic differential equations with jumps are introduced as a generalization of previous ones. Their respective order of convergence is obtained as well.
\end{abstract}

Keywords: Jump diffusion process; stochastic differential equation; numerical integration; local linearization; weak convergence

2000 Mathematics Subject Classification: Primary 60J75; 60H35

Secondary $60 \mathrm{~J} 60$

\section{Introduction}

In a number of problems in mathematical physics, biology, economics, finance, and other fields the estimation of functionals of jump diffusion processes plays a prominent role; see [4], [5], [15], and [23]. In particular, the jump diffusion processes defined through stochastic differential equations (SDEs) have become an important mathematical tool for describing the dynamics of several phenomena, for example, the dynamic of assets prices in the market, the firing of neurons, and so on. Since exact representation for functionals of these processes is only possible in a few cases, approximate representations are required. Different types of such weak approximations have already been proposed in [2], [6], [11], [12], [13], and [19], which are essentially based on Itô-Taylor expansions of the jump diffusion process. The weak convergence properties of the approximations based on these expansions as well as their numerical instability for a number of nonlinear SDEs have also been studied; see [3] and [14].

The main aim of this paper is to investigate another kind of weak approximation for SDEs with jumps: the weak local linear (WLL) approximation. In the framework of ordinary SDEs (without jumps), the WLL approximations have recently been proposed as stable alternatives to the abovementioned conventional approximations based on Itô-Taylor expansions (see [3], [14], [16], and [21]), and they have been key in the derivation of effective inference methods for SDEs (see [20], [21], and [22]) and for continuous-discrete space-state models (see [7], [8], and [17]). Therefore, the present study is well motivated.

Specifically, in this note the WLL approximations for SDEs are extended to the more general case of equations with jumps, and their rate of weak convergence is studied.

Received 21 September 2007; revision received 2 December 2007.

* Current address: The Department of Mathematics and Statistics, McGill University, 805 Sherbrooke Street West, Montreal, Quebec, Canada, H3A 2K6. Email address: carbonell@math.mcgill.ca

** Postal address: Departamento de Matemática Interdisciplinaria, Instituto de Cibernética, Matemática y Física, Calle 15, No. 551, e/C y D, Vedado, La Habana 4, C.P. 10400, Cuba. 


\section{WLL approximations}

Let $(\Omega, \mathcal{F}, \mathrm{P})$ be the underlying complete probability space, and let $\left\{\mathcal{F}_{t}, t \geq t_{0}\right\}$ be an increasing right-continuous family of complete sub- $\sigma$-algebras of $\mathcal{F}$. Consider a $d$-dimensional jump diffusion process $\boldsymbol{x}$ defined by the following SDE:

$$
\begin{gathered}
\mathrm{d} \boldsymbol{x}(t)=\boldsymbol{f}(t, \boldsymbol{x}(t)) \mathrm{d} t+\boldsymbol{G}(t) \mathrm{d} \boldsymbol{w}(t)+\sum_{i=1}^{p} \boldsymbol{h}_{i}(t, \boldsymbol{x}(t)) \mathrm{d} \boldsymbol{q}^{i}(t), \quad t \in\left[t_{0}, T\right], T<\infty, \\
\boldsymbol{x}\left(t_{0}\right)=\boldsymbol{x}_{0} .
\end{gathered}
$$

Here, $\boldsymbol{w}$ is an $m$-dimensional $\mathscr{F}_{t}$-adapted standard Wiener process and each $\boldsymbol{q}^{i}, i=1, \ldots, p$, could be either an $\mathcal{F}_{t}$-adapted Poisson counting process $\boldsymbol{n}^{i}$ with intensity $\mu^{i}$ or an $\mathcal{F}_{t}$-adapted compensated Poisson processes $\left\{\boldsymbol{n}^{i}(t)-\mu^{i} t: t \geq t_{0}\right\}$. Also, $\boldsymbol{f}, \boldsymbol{h}_{i}: \mathbb{R} \times \mathbb{R}^{d} \rightarrow \mathbb{R}^{d}$ and $\boldsymbol{G}: \mathbb{R} \rightarrow \mathbb{R}^{d} \times \mathbb{R}^{m}$ are functions satisfying linear growth restriction, and uniform Lipschitz and smoothness conditions that ensure the existence and uniqueness of a solution to (1). It is also assumed that $\boldsymbol{w}$ and $\boldsymbol{q}^{j}$ are all independent with zero probability of simultaneous jumps.

Let $(t)_{\delta}=\left\{t_{0} \leq t_{1} \leq \cdots \leq t_{n}<\cdots<\infty\right\}$ be a time partition defined as a sequence of $\mathcal{F}_{t_{n}}$-measurable stopping times $t_{n}, n=0,1, \ldots$, that satisfy

$$
\sup _{n}\left(\delta_{n}\right) \leq \delta<1 \quad \text { with probability } 1,
$$

where $\delta_{n}=t_{n+1}-t_{n}$, and define

$$
n_{t}:=\max \left\{n=0,1,2, \ldots: t_{n} \leq t\right\}<\infty .
$$

\subsection{WLL discretizations for SDEs}

Let us consider the $d$-dimensional diffusion process $z$ defined by the SDE

$$
\begin{gathered}
\mathrm{d} \boldsymbol{z}(t)=\overline{\boldsymbol{f}}(t, \boldsymbol{z}(t)) \mathrm{d} t+\boldsymbol{G}(t) \mathrm{d} \boldsymbol{w}(t) \quad \text { for } t \in[a, b], \\
\boldsymbol{z}(a)=z_{0},
\end{gathered}
$$

where $\overline{\boldsymbol{f}}$ is a differentiable function, $\boldsymbol{w}$ and $\boldsymbol{G}$ are defined as in (1), and $t_{0} \leq a \leq b \leq T$.

Definition 1. ([3].) For a given time discretization $(t)_{\delta}$, the order- $(\beta=1,2)$ WLL discretization of the diffusion process $z$ is defined by the recurrent relation

$$
\boldsymbol{y}_{t_{n+1}}=\boldsymbol{y}_{t_{n}}+\boldsymbol{\phi}_{\beta}\left(t_{n}, \boldsymbol{y}_{t_{n}} ; t_{n+1}-t_{n}\right)+\boldsymbol{\eta}\left(t_{n}, \boldsymbol{y}_{t_{n}} ; t_{n+1}-t_{n}\right),
$$

where $\boldsymbol{y}_{t_{0}}=z_{0}$,

$$
\begin{gathered}
\boldsymbol{\phi}_{\beta}(t, \boldsymbol{y} ; \delta)=\int_{0}^{\delta} \exp \left(\frac{\partial \overline{\boldsymbol{f}}(t, \boldsymbol{y})}{\partial \boldsymbol{y}}(\delta-s)\right)\left(\overline{\boldsymbol{f}}(t, \boldsymbol{y})+\overline{\boldsymbol{b}}_{\beta}(t, \boldsymbol{y}) s\right) \mathrm{d} s, \\
\overline{\boldsymbol{b}}_{\beta}(t, \boldsymbol{y})= \begin{cases}\frac{\partial \overline{\boldsymbol{f}}(t, \boldsymbol{y})}{\partial t}, & \beta=1, \\
\frac{\partial \overline{\boldsymbol{f}}(t, \boldsymbol{y})}{\partial t}+\frac{1}{2} \sum_{k, l=1}^{d}\left(\boldsymbol{G}(t) \boldsymbol{G}^{\top}(t)\right)^{k, l} \frac{\partial^{2} \overline{\boldsymbol{f}}(t, \boldsymbol{y})}{\partial \boldsymbol{y}^{k} \partial \boldsymbol{y}^{l}}, & \beta=2,\end{cases}
\end{gathered}
$$


for all $(t, \boldsymbol{y}) \in \mathbb{R} \times \mathbb{R}^{d}$ and $\delta>0$, and $\boldsymbol{\eta}(t, \boldsymbol{y} ; \delta)$ is a zero-mean Gaussian process with variance matrix

$$
\boldsymbol{\Sigma}(t, \boldsymbol{y} ; \delta)=\int_{0}^{\delta} \exp \left(\frac{\partial \overline{\boldsymbol{f}}(t, \boldsymbol{y})}{\partial \boldsymbol{y}}(\delta-s)\right) \boldsymbol{G}(t+s) \boldsymbol{G}^{\top}(t+s) \exp \left(\left(\frac{\partial \overline{\boldsymbol{f}}(t, \boldsymbol{y})}{\partial \boldsymbol{y}}\right)^{\top}(\delta-s)\right) \mathrm{d} s .
$$

On the basis of this discretization, various weak numerical integrators and inference methods for SDEs have been proposed (see [3] and [9] for an updated review), which differ in the way that they compute the integrals in (4) and (5).

Definition 2. ([3].) For a given time discretization $(t)_{\delta}$, the stochastic process $\boldsymbol{y}_{\beta}^{\delta}=\left\{\boldsymbol{y}_{\beta}^{\delta}(t)\right.$, $\left.t \in\left[t_{0}, T\right]\right\}$ is called the order- $(\beta=1,2)$ WLL approximation of the diffusion process $z$ if

$$
\boldsymbol{y}_{\beta}^{\delta}(t)=\boldsymbol{y}_{t_{n_{t}}}+\boldsymbol{\phi}_{\beta}\left(t_{n_{t}}, \boldsymbol{y}_{t_{n_{t}}} ; t-t_{n_{t}}\right)+\boldsymbol{\eta}\left(t_{n_{t}}, \boldsymbol{y}_{t_{n_{t}}} ; t-t_{n_{t}}\right),
$$

where $\left\{\boldsymbol{y}_{t_{n}}\right\}, n=0,1, \ldots$, is the WLL discretization (3).

Note that the WLL approximation (6) is a continuous-time stochastic process that coincides with the WLL discretization (3) at each discretization time $t_{n} \in(t)_{\delta}$.

It is also convenient to remark that the WLL approximation (6) coincides with the weak solution to the piecewise SDE

$$
\begin{gathered}
\mathrm{d} \boldsymbol{y}(t)=\overline{\boldsymbol{p}}_{\beta}\left(t, \boldsymbol{y}(t) ; t_{n}, \boldsymbol{y}\left(t_{n}\right)\right) \mathrm{d} s+\boldsymbol{G}(t) \mathrm{d} \boldsymbol{w}(t), \quad t \in\left[t_{n}, t_{n+1}\right], n=0,1, \ldots, n_{T}-1, \\
\boldsymbol{y}\left(t_{n}\right)=\boldsymbol{y}_{t_{n}},
\end{gathered}
$$

where the function $\overline{\boldsymbol{p}}_{\beta}$ is defined as

$$
\overline{\boldsymbol{p}}_{\beta}(s, \boldsymbol{v} ; r, \boldsymbol{u})=\overline{\boldsymbol{f}}(r, \boldsymbol{u})+\frac{\partial \overline{\boldsymbol{f}}(r, \boldsymbol{u})}{\partial \boldsymbol{u}}(\boldsymbol{v}-\boldsymbol{u})+\overline{\boldsymbol{b}}_{\beta}(r, \boldsymbol{u})(s-r)
$$

for all $\boldsymbol{v}, \boldsymbol{u} \in \mathbb{R}^{d}$ and $s, r \in \mathbb{R}, s>r$, which, for $\beta=1$ and $\beta=2$, is just the first-order Taylor and Itô-Taylor expansions of $\overline{\boldsymbol{f}}$, respectively; see [3].

\subsection{WLL discretizations for SDEs with jumps}

Consider the sequence of jump times $\{\boldsymbol{\sigma}\}_{\mu^{i}}=\left\{\sigma_{i, n}: n=0,1,2, \ldots\right\}$ associated to $\boldsymbol{q}^{i}$, which is defined as an increasing sequence of random variables such that $\sigma_{i, n+1}-\sigma_{i, n}$ is exponentially distributed with parameter $\mu^{i}$ for all $n$ and $i$. Without loss of generality, we can assume that $\{\sigma\}_{\mu^{i}} \subset(t)_{\delta} \subset\left[t_{0}, T\right]$ for all $i=1, \ldots, p$. In addition, let us assume that only the first $r$ Poisson processes $\boldsymbol{q}^{i}$ are compensated.

It is well known that (see [18]) the solution to (1) is given by

$$
\boldsymbol{x}(t)=\boldsymbol{x}(t-)+\sum_{i=1}^{p} \boldsymbol{h}_{i}(t, \boldsymbol{x}(t-)) \Delta \boldsymbol{n}_{t}^{i},
$$

where $\Delta \boldsymbol{n}_{t}^{i}$ is the increment of the process $\boldsymbol{n}^{i}$ at the time instant $t$ and $\boldsymbol{x}(t-)$ denotes the solution to the $\operatorname{SDE}(2)$ with

$$
\overline{\boldsymbol{f}}(t, \boldsymbol{z}(t))=\boldsymbol{f}(t, \boldsymbol{z}(t))-\sum_{i=1}^{r} \boldsymbol{h}_{i}(t, \boldsymbol{z}(t)) \mu^{i},
$$

and initial condition $\boldsymbol{z}\left(\sigma_{i, n}\right)=\boldsymbol{x}\left(\sigma_{i, n}\right)$ for all $t$ between two consecutive jump times $\sigma_{i, n}$ and $\sigma_{j, m}$.

The above leads to the following two definitions. 
Definition 3. For a given time discretization $(t)_{\delta}$, the order- $(\beta=1,2)$ WLL discretization of the jump diffusion process $\boldsymbol{x}$ is recursively defined by

$$
\boldsymbol{y}_{t_{n+1}}=\boldsymbol{y}_{t_{n+1}-}+\sum_{i=1}^{p} \boldsymbol{h}_{i}\left(t_{n+1}, \boldsymbol{y}_{t_{n+1}-}\right) \Delta \boldsymbol{n}_{t_{n+1}}^{i}
$$

where

$$
\boldsymbol{y}_{t_{n+1}-}=\boldsymbol{y}_{t_{n}}+\boldsymbol{\phi}_{\beta}\left(t_{n}, \boldsymbol{y}_{t_{n}} ; t_{n+1}-t_{n}\right)+\boldsymbol{\eta}\left(t_{n}, \boldsymbol{y}_{t_{n}} ; t_{n+1}-t_{n}\right)
$$

denotes the WLL discretization of (2) with $\overline{\boldsymbol{f}}$ defined as in (8).

Definition 4. For a given time discretization $(t)_{\delta}$, the stochastic process $\boldsymbol{y}_{\beta}^{\delta}=\left\{\boldsymbol{y}_{\beta}^{\delta}(t), t \in\right.$ $\left.\left[t_{0}, T\right]\right\}$ is called the order- $(\beta=1,2)$ WLL approximation of the jump diffusion process $\boldsymbol{x}$ if

$$
\boldsymbol{y}_{\beta}^{\delta}(t)=\boldsymbol{y}_{\beta}^{\delta}(t-)+\sum_{i=1}^{p} \boldsymbol{h}_{i}\left(t, \boldsymbol{y}_{\beta}^{\delta}(t-)\right) \Delta \boldsymbol{n}_{t}^{i}
$$

where

$$
\boldsymbol{y}_{\beta}^{\delta}(t-)=\boldsymbol{y}_{t_{n_{t}}}+\boldsymbol{\phi}_{\beta}\left(t_{n_{t}}, \boldsymbol{y}_{t_{n_{t}}} ; t-t_{n_{t}}\right)+\boldsymbol{\eta}\left(t_{n_{t}}, \boldsymbol{y}_{t_{n_{t}}} ; t-t_{n_{t}}\right)
$$

denotes the WLL approximation of (2) with $\bar{f}$ defined as in (8).

\section{Convergence of WLL approximations}

Let $\mathcal{M}$ be the set of multi-indices $\alpha=\left(j_{1}, \ldots, j_{l(\alpha)}\right), j_{i} \in\{0,1, \ldots, m\}$, and $i=$ $1, \ldots, l(\alpha)$, where $l(\alpha)$ denotes the length of the multi-index $\alpha$. Denote by $-\alpha$ and $\alpha-$ the multi-indices in $\mathcal{M}$ that are obtained by deleting the first and the last component of $\alpha$, respectively. The multi-index of length 0 will be denoted by $\nu$. Let $\Gamma_{\beta} \subset \mathcal{M}, \beta=1,2$, be the hierarchical set

$$
\Gamma_{\beta}=\{\alpha \in \mathcal{M}: l(\alpha) \leq \beta\},
$$

and let $\mathscr{B}\left(\Gamma_{\beta}\right)$ be the remainder set of $\Gamma_{\beta}$,

$$
\mathscr{B}\left(\Gamma_{\beta}\right)=\{\alpha \in \mathcal{M}: l(\alpha)=\beta+1\} .
$$

Denote by $\mathscr{H}_{v}, \mathscr{H}_{(0)}$, and $\mathscr{H}_{(1)}$ the sets of the adapted right-continuous process $h=\{h(t), t \geq$ $0\}$ on $(\Omega, \mathcal{F}, \mathrm{P})$ with left-hand limits that satisfy

$$
|h(t)|<\infty, \quad \int_{0}^{t}|h(s)| \mathrm{d} s<\infty, \quad \text { and } \quad \int_{0}^{t}|h(s)|^{2} \mathrm{~d} s<\infty, \quad \text { with probability } 1,
$$

respectively. In addition, define $\mathscr{H}_{(j)}=\mathscr{H}_{(1)}$ for $j=2, \ldots, m$ and $m \geq 2$. Then, for $\alpha=\left(j_{1}, \ldots, j_{l(\alpha)}\right), l(\alpha) \geq 2$, recursively define the set $\mathscr{H}_{\alpha}$ as the totality of adapted rightcontinuous processes $h$ with left-hand limits such that $\left\{I_{\alpha-}[h(\cdot)]_{\rho, t}, t \geq 0\right\} \in \mathscr{H}_{j_{l(\alpha)}}$, where, for $h \in \mathscr{H}_{\alpha}$, the multiple Itô integral $I_{\alpha}[h(\cdot)]_{\rho, \tau}$ is recursively defined by

$$
I_{\alpha}[h(\cdot)]_{\rho, \tau}:= \begin{cases}h(\tau), & l(\alpha)=0 \\ \int_{\rho}^{\tau} I_{\alpha-}[h(\cdot)]_{\rho, s} \mathrm{~d} w_{s}^{j_{l(\alpha)}}, & l(\alpha) \geq 1 .\end{cases}
$$


Let

$$
L^{0}=\frac{\partial}{\partial t}+\sum_{k=1}^{d} \overline{\boldsymbol{f}}^{k} \frac{\partial}{\partial \boldsymbol{x}^{k}}+\frac{1}{2} \sum_{k, l=1}^{d} \sum_{i=1}^{m} \boldsymbol{G}^{k, i} \boldsymbol{G}^{l, i} \frac{\partial^{2}}{\partial \boldsymbol{x}^{k} \partial \boldsymbol{x}^{l}}
$$

be the diffusion operator of the SDE (2), and define

$$
L^{j}=\sum_{k=1}^{d} \boldsymbol{G}^{k, j} \frac{\partial}{\partial \boldsymbol{x}^{k}}, \quad j=1, \ldots, m .
$$

Then, for the hierarchical set $\Gamma_{\beta}$ and any two stopping times $\rho$ and $\tau$ satisfying $0 \leq \rho \leq \tau \leq T$, the expression

$$
\boldsymbol{x}(\tau)=\boldsymbol{x}(\tau-)+\sum_{i=1}^{p} \boldsymbol{h}_{i}(\tau, \boldsymbol{x}(\tau-)) \Delta \boldsymbol{n}_{t}^{i}
$$

with

$$
\boldsymbol{x}(\tau-)=\boldsymbol{x}(\rho)+\sum_{\alpha \in \Gamma_{\beta} \backslash\{v\}} I_{\alpha}\left[\lambda_{\alpha}(\rho, \boldsymbol{x}(\rho))\right]_{\rho, \tau}+\sum_{\alpha \in \mathcal{B}\left(\Gamma_{\beta}\right)} I_{\alpha}\left[\lambda_{\alpha}(\cdot, \boldsymbol{x}(\cdot))\right]_{\rho, \tau},
$$

provides the weak Itô-Taylor expansion of the jump diffusion process solution $x$ of the SDE (1), where

$$
\lambda_{\alpha}= \begin{cases}L^{j_{1}} \cdots L^{j_{l(\alpha)-1}} \overline{\boldsymbol{f}}, & j_{l(\alpha)}=0, \\ L^{j_{1}} \cdots L^{j_{l(\alpha)-1}} \boldsymbol{G}^{j_{l(\alpha)},} & j_{l(\alpha)} \neq 0,\end{cases}
$$

denotes the Itô coefficient function for each $\alpha$, and $\boldsymbol{G}^{j}$ is the $j$ th column vector of $\boldsymbol{G}$.

Furthermore, denote by $\mathcal{C}_{\mathcal{P}}^{l}\left(\mathbb{R}^{d}, \mathbb{R}\right)$ the space of $l$ time-continuously differentiable functions $g: \mathbb{R}^{d} \rightarrow \mathbb{R}$ for which $g$ and all its partial derivatives up to order $l$ have polynomial growth. For $l=1,2, \ldots$, define $P_{l}=\left\{\boldsymbol{p} \in\{1, \ldots, d\}^{l}\right\}$ and, for each $\boldsymbol{p}=\left(p_{1}, \ldots, p_{l}\right) \in P_{l}$, define the function $\boldsymbol{F}_{\boldsymbol{p}}: \mathbb{R}^{d} \rightarrow \mathbb{R}$ as

$$
\boldsymbol{F}_{\boldsymbol{p}}(\boldsymbol{x})=\prod_{i=1}^{l} \boldsymbol{x}^{p_{i}}
$$

The following lemma provides general conditions to assure that a discrete-time approximation $\boldsymbol{u}^{\delta}$ converges weakly to $\boldsymbol{x}$.

Lemma 1. ([1, Theorem 10.7.1].) Let $\boldsymbol{u}^{\delta}$ be a discrete-time approximation of the process $\boldsymbol{x}$ (the solution to $(1))$ corresponding to a time discretization $(t)_{\delta}$, and assume that

$$
\begin{gathered}
\mathrm{E}\left(\left|\boldsymbol{x}_{0}\right|^{i}\right)<\infty \text { for } i=1,2, \ldots, \\
\left|\mathrm{E}\left(g\left(\boldsymbol{x}_{0}\right)\right)-\mathrm{E}\left(g\left(\boldsymbol{u}_{0}^{\delta}\right)\right)\right| \leq C_{0} \delta^{\beta}
\end{gathered}
$$

for any $g \in \mathcal{C}_{\mathcal{P}}^{2(\beta+1)}\left(\mathbb{R}^{d}, \mathbb{R}\right)$, where $C_{0}$ is a positive constant. Suppose that

$$
\boldsymbol{f}^{k}, \boldsymbol{G}^{k, j}, \boldsymbol{h}_{l}^{k} \in \mathcal{C}_{\mathcal{P}}^{2(\beta+1)}\left(\left[t_{0}, T\right] \times \mathbb{R}^{d}, \mathbb{R}\right)
$$

for all $k=1, \ldots, d, j=1, \ldots, m$, and $l=1, \ldots, p$, and suppose that the Itô coefficient functions $\lambda_{\alpha}$ satisfy linear growth bounds for all $\alpha \in \Gamma_{\beta}$. In addition, suppose that, for each 
$q=1,2, \ldots$, there exist constants $K<\infty$ and $r \in \mathbb{N}^{+}$, which do not depend on $\delta$, such that

$$
\begin{gathered}
\mathrm{E}\left(\max _{0 \leq n \leq n_{T}}\left|\boldsymbol{u}_{t_{n}-}^{\delta}\right|^{2 q} \mid \mathcal{F}_{t_{0}}\right) \leq K\left(1+\left|\boldsymbol{u}_{0}^{\delta}\right|^{2 r}\right), \\
\mathrm{E}\left(\left|\boldsymbol{u}_{t_{n+1}-}^{\delta}-\boldsymbol{u}_{t_{n}}^{\delta}\right|^{2 q} \mid \mathcal{F}_{t_{n}}\right) \leq K\left(1+\max _{0 \leq k \leq n}\left|\boldsymbol{u}_{t_{k}}^{\delta}\right|^{2 r}\right)\left(t_{n+1}-t_{n}\right)^{q},
\end{gathered}
$$

and

$$
\begin{aligned}
& \left|\mathrm{E}\left(\boldsymbol{F}_{\boldsymbol{p}}\left(\boldsymbol{u}_{t_{n+1}-}^{\delta}-\boldsymbol{u}_{t_{n}}^{\delta}\right)-\boldsymbol{F}_{\boldsymbol{p}}\left(\sum_{\alpha \in \Gamma_{\beta} \backslash\{v\}} I_{\alpha}\left[\lambda_{\alpha}\left(t_{n}, \boldsymbol{u}_{t_{n}}^{\delta}\right)\right]_{t_{n}, t_{n+1}}\right) \mid \mathcal{F}_{t_{n}}\right)\right| \\
& \leq K\left(1+\max _{0 \leq k \leq n}\left|\boldsymbol{u}_{t_{k}}^{\delta}\right|^{2 r}\right)\left(t_{n+1}-t_{n}\right) \delta^{\beta},
\end{aligned}
$$

for all $n=0,1, \ldots, n_{T}-1, l=1, \ldots, 2 \beta+1, p \in P_{l}$, and $t_{n}, t_{n+1}(t)_{\delta}$. Then we obtain

$$
\left|\mathrm{E}\left(g\left(\boldsymbol{x}_{T}\right)\right)-\mathrm{E}\left(g\left(\boldsymbol{u}_{T}^{\delta}\right)\right)\right| \leq C_{g} \delta^{\beta}
$$

for some positive constant $C_{g}$.

The main result of this section is stated in the next theorem. It establishes the weak convergence of the WLL approximation (10) to the jump diffusion process $\boldsymbol{x}$. Its proof will be based on demonstrating that the WLL approximation $\boldsymbol{y}_{\beta}^{\delta}$ satisfies the conditions of Lemma 1.

Theorem 1. Suppose that

$$
\begin{gathered}
\mathrm{E}\left(\left|x_{0}\right|^{j}\right)<\infty \quad \text { for } j=1,2, \ldots, \\
\left|\mathrm{E}\left(g\left(\boldsymbol{x}_{0}\right)\right)-\mathrm{E}\left(g\left(\boldsymbol{y}_{\beta}^{\delta}\left(t_{0}\right)\right)\right)\right| \leq C_{0} \delta^{\beta}
\end{gathered}
$$

for some $C_{0}>0$ and all $g \in \mathcal{C}_{\mathcal{P}}^{2(\beta+1)}\left(\mathbb{R}^{d}, \mathbb{R}\right)$. Also assume that condition (12) holds, and let $\widetilde{K}$ be a positive constant such that

$$
\begin{gathered}
|\boldsymbol{f}(t, \boldsymbol{x})|+|\boldsymbol{G}(t)| \leq \widetilde{K}(1+|\boldsymbol{x}|), \\
\left|\frac{\partial \boldsymbol{f}(t, \boldsymbol{x})}{\partial t}\right|+\left|\frac{\partial \boldsymbol{f}(t, \boldsymbol{x})}{\partial \boldsymbol{x}}\right|+\left|\frac{\partial^{2} \boldsymbol{f}(t, \boldsymbol{x})}{\partial \boldsymbol{x}^{2}}\right| \leq \widetilde{K} \\
\left|\boldsymbol{h}_{i}(t, \boldsymbol{x})\right| \leq \widetilde{K}(1+|\boldsymbol{x}|), \quad i=1, \ldots, p,
\end{gathered}
$$

for all $t \in\left[t_{0}, T\right]$ and $\boldsymbol{x} \in \mathbb{R}^{d}$. Then there exists a positive constant $C_{g}$ such that the WLL approximation $\boldsymbol{y}_{\beta}^{\delta}$ satisfies

$$
\left|\mathrm{E}(g(\boldsymbol{x}(T)))-\mathrm{E}\left(g\left(\boldsymbol{y}_{\beta}^{\delta}(T)\right)\right)\right| \leq C_{g} \delta^{\beta} .
$$

In order to prove Theorem 1, the following two lemmas will be needed. Lemma 2, below, presents known results on WLL approximations for ordinary SDEs, while Lemma 3, below, extends these results to the WLL approximations for ordinary SDEs with jumps.

Lemma 2. ([3, Lemmas 6 and 7].) Let $\boldsymbol{y}_{\beta}^{\delta}$ be the WLL approximation (6) to the solution of the ordinary SDE (2). Then, under the assumptions of Theorem 1, we have

$$
\mathrm{E}\left(\sup _{a \leq t \leq b}\left|\boldsymbol{y}_{\beta}^{\delta}(t)\right|^{2 q} \mid \mathcal{F}_{a}\right) \leq K_{1}\left(1+\left|\boldsymbol{y}_{\beta}^{\delta}(a)\right|^{2 q}\right),
$$


where $K_{1}$ is a positive constant for each $q=1,2, \ldots$ Moreover, if

$$
z_{\beta}^{\delta}(t)=\boldsymbol{y}_{t_{n_{t}}}+\sum_{\alpha \in \Gamma_{\beta} \backslash\{v\}} I_{\alpha}\left[\Lambda_{\alpha}\left(t_{n_{t}}, \boldsymbol{y}_{t_{t}} ; t_{n_{t}}, \boldsymbol{y}_{t_{n_{t}}}\right)\right]_{t_{n_{t}}, t}+\sum_{\alpha \in \mathcal{B}\left(\Gamma_{\beta}\right)} I_{\alpha}\left[\Lambda_{\alpha}\left(\cdot, \boldsymbol{y} ; t_{n_{t}}, \boldsymbol{y}_{t_{n_{t}}}\right)\right]_{t_{n_{t}}, t}
$$

denotes the Itô-Taylor expansion of the solution to (7) with Itô coefficient function

$$
\Lambda_{\alpha}(s, \boldsymbol{v} ; r, \boldsymbol{u})= \begin{cases}L^{j_{1}} \cdots L^{j_{l(\alpha)-1}} \overline{\boldsymbol{p}}_{\beta}(s, \boldsymbol{v} ; r, \boldsymbol{u}), & j_{l(\alpha)}=0 \\ L^{j_{1}} \cdots L^{j_{l(\alpha)-1}} \boldsymbol{G}^{j_{l(\alpha)},} & j_{l(\alpha)} \neq 0\end{cases}
$$

then

$$
\boldsymbol{y}_{\beta}^{\delta} \equiv z_{\beta}^{\delta}
$$

and

$$
I_{\alpha}\left[\Lambda_{\alpha}\left(t_{n_{t}}, \boldsymbol{y}_{t_{n_{t}}} ; t_{n_{t}}, \boldsymbol{y}_{t_{n_{t}}}\right)\right]_{t_{n_{t}}, t}=I_{\alpha}\left[\lambda_{\alpha}\left(t_{n_{t}}, \boldsymbol{y}_{t_{n_{t}}}\right)\right]_{t_{n_{t}}, t}
$$

hold for all $\alpha \in \Gamma_{\beta} \backslash\{v\}$ and $t \in[a, b]$, where $\lambda_{\alpha}$ is the Itô coefficient function defined in (11).

Lemma 3. Let $\boldsymbol{y}_{\beta}^{\delta}$ be the WLL approximation (10) to the solution of the SDE with jumps (1), and let $z_{\beta}^{\delta}=\left\{z_{\beta}^{\delta}(t), t \in\left[t_{0}, T\right]\right\}$ be the stochastic process defined as

$$
\boldsymbol{z}_{\beta}^{\delta}(t)=\boldsymbol{z}_{\beta}^{\delta}(t-)+\sum_{i=1}^{p} \boldsymbol{h}_{i}\left(t, \boldsymbol{z}_{\beta}^{\delta}(t-)\right) \Delta \boldsymbol{n}_{t}^{i}
$$

where $z_{\beta}^{\delta}(t-)$ denotes the Itô-Taylor expansion (17). Then, under the assumptions of Theorem 1, we find that

$$
\mathrm{E}\left(\sup _{t_{0} \leq t \leq T}\left|\boldsymbol{y}_{\beta}^{\delta}(t)\right|^{2 q} \mid \mathcal{F}_{t_{0}}\right) \leq K_{2}\left(1+\left|\boldsymbol{y}_{0}\right|^{2 q}\right)
$$

and

$$
\boldsymbol{y}_{\beta}^{\delta} \equiv z_{\beta}^{\delta}
$$

hold, where $K_{2}$ is a positive constant.

Proof. Let $N_{T}=\sum_{i=1}^{p} \boldsymbol{n}^{i}(T)$ denote the total number of jumps up to time $T$, and let $\{t\}_{N_{T}}=\left\{t_{j}: j=0, \ldots, N_{T}\right\}$ denote a sequence of time instants such that $\{t\}_{N_{T}} \subset(\tau)_{\delta}$, $t_{j} \in\left\{\tau_{0} \cup\{\boldsymbol{\sigma}\}_{\mu^{1}} \cup \cdots \cup\{\boldsymbol{\sigma}\}_{\mu^{p}}\right\}$, and $t_{j}<t_{j+1}$, for all $j=0, \ldots, N_{T}-1$. Furthermore, let $Z_{s}=\left\{\boldsymbol{n}^{i}\left(t_{j}\right): t_{j} \leq s, t_{j} \in\{t\}_{N_{T}}, i=1, \ldots, p\right\}$ for $s \geq t_{0}$. By defining

$$
e_{j}=\mathrm{E}\left(\sup _{t_{0} \leq s \leq t_{j}}\left|\boldsymbol{y}_{\beta}^{\delta}(s)\right|^{2 q} \mid \mathcal{F}_{t_{0}} ; Z_{t_{j}}\right)
$$

with $t_{j} \in\{t\}_{N_{T}}$, we find that

$$
e_{j+1} \leq e_{j}+\Delta e_{j+1}
$$


where $\Delta e_{j+1}=\mathrm{E}\left(\sup _{t_{j}<s \leq t_{j+1}}\left|\boldsymbol{y}_{\beta}^{\delta}(s)\right|^{2 q} \mid \mathcal{F}_{t_{0}} ; Z_{t_{j+1}}\right)$. From (10) and (16), we obtain

$$
\begin{aligned}
\Delta e_{j+1} \leq(p+1)^{2 q-1} & \left(\mathrm{E}\left(\sup _{t_{j}<s \leq t_{j+1}}\left|\boldsymbol{y}_{\beta}^{\delta}(s-)\right|^{2 q} \mid \mathcal{F}_{t_{0}} ; Z_{t_{j+1}}\right)\right. \\
& \left.+\sum_{i=1}^{p} \mathrm{E}\left(\sup _{t_{j}<s \leq t_{j+1}}\left|\boldsymbol{h}_{i}\left(s, \boldsymbol{y}_{\beta}^{\delta}(s-)\right) \Delta \boldsymbol{n}_{s}^{i}\right|^{2 q} \mid \widetilde{F}_{t_{0}} ; Z_{t_{j+1}}\right)\right) \\
\leq(p+1)^{2 q-1}( & \left(1+2^{2 q-1} p \widetilde{K}^{2 q}\right) \mathrm{E}\left(\sup _{t_{j} \leq s \leq t_{j+1}}\left|\boldsymbol{y}_{\beta}^{\delta}(s-)\right|^{2 q} \mid \mathcal{F}_{t_{0}} ; Z_{t_{j+1}}\right) \\
& \left.+2^{2 q-1} p \widetilde{K}^{2 q}\right) .
\end{aligned}
$$

By definition, for all $s \in\left[t_{j}, t_{j+1}\right], \boldsymbol{y}_{\beta}^{\delta}(s-)$ is the WLL approximation to the solution of an ordinary SDE (without jumps). Therefore, by using Lemma 2 in that time interval, it follows that

$$
\begin{aligned}
\mathrm{E}\left(\sup _{t_{j} \leq s \leq t_{j+1}}\left|\boldsymbol{y}_{\beta}^{\delta}(s-)\right|^{2 q} \mid \mathcal{F}_{t_{0}} ; Z_{t_{j+1}}\right) & =\mathrm{E}\left(\mathrm{E}\left(\sup _{t_{j} \leq s \leq t_{j+1}}\left|\boldsymbol{y}_{\beta}^{\delta}(s-)\right|^{2 q} \mid \mathcal{F}_{t_{j}}\right) \mid \mathcal{F}_{t_{0}} ; Z_{t_{j+1}}\right) \\
& \leq K_{1}\left(1+\mathrm{E}\left(\left|\boldsymbol{y}_{\beta}^{\delta}\left(t_{j}\right)\right|^{2 q} \mid \mathcal{F}_{t_{0}} ; Z_{t_{j+1}}\right)\right) \\
& \leq K_{1}\left(1+\mathrm{E}\left(\sup _{t_{0} \leq s \leq t_{j}}\left|\boldsymbol{y}_{\beta}^{\delta}\left(t_{j}\right)\right|^{2 q} \mid \mathcal{F}_{t_{0}} ; Z_{t_{j}}\right)\right) .
\end{aligned}
$$

Thus,

$$
\Delta e_{j+1} \leq C_{1} e_{j}+C_{2},
$$

where $C_{1}=(p+1)^{2 q-1}\left(1+2^{2 q-1} p \widetilde{K}^{2 q}\right) K_{1}$ and $C_{2}=C_{1}+2^{2 q-1} p \widetilde{K}^{2 q}$. In this way we obtain

$$
e_{j+1} \leq\left(1+C_{1}\right) e_{j}+C_{2}
$$

which implies that

$$
\begin{aligned}
e_{j+1} & \leq\left(1+C_{1}\right)^{j+1} e_{0}+\frac{C_{2}}{C_{1}}\left(\left(1+C_{1}\right)^{j}-1\right) \\
& \leq\left(1+C_{1}\right)^{j+1}\left(e_{0}+\frac{C_{2}}{C_{1}}\right) \\
& \leq \frac{C_{2}}{C_{1}}\left(1+C_{1}\right)^{j+1}\left(1+e_{0}\right) .
\end{aligned}
$$

By using the above inequality and taking $j=N_{T}$, we have

$$
\mathrm{E}\left(\sup _{t_{0} \leq s \leq T}\left|\boldsymbol{y}_{\beta}^{\delta}(s)\right|^{2 q} \mid \mathcal{F}_{t_{0}} ; Z_{t_{N_{T}}}\right) \leq \frac{C_{2}}{C_{1}}\left(1+C_{1}\right)^{1+N_{T}}\left(1+\left|\boldsymbol{y}_{\beta}^{\delta}\left(t_{0}\right)\right|^{2 q}\right) .
$$

By taking into account the fact that

$$
\mathrm{E}\left(\kappa^{N_{T}}\right)=\mathrm{E}\left(\exp \left(N_{T} \ln (\kappa)\right)\right)=\exp \left((\kappa-1)\left(T-t_{0}\right) \sum_{i=1}^{p} \mu^{i}\right)
$$


for any constant $\kappa>1$, it follows that

$$
\begin{aligned}
\mathrm{E}\left(\sup _{t_{0} \leq s \leq T}\left|\boldsymbol{y}_{\beta}^{\delta}(s)\right|^{2 q} \mid \mathcal{F}_{t_{0}}\right) & =\mathrm{E}\left(\mathrm{E}\left(\sup _{t_{0} \leq s \leq T}\left|\boldsymbol{y}_{\beta}^{\delta}(s)\right|^{2 q} \mid \widetilde{F}_{t_{0}} ; Z_{t_{N_{T}}}\right) \mid \mathcal{F}_{t_{0}}\right) \\
& \leq \frac{C_{2}}{C_{1}}\left(1+C_{1}\right) \exp \left(C_{1}\left(T-t_{0}\right) \sum_{i=1}^{p} \mu^{i}\right)\left(1+\left|\boldsymbol{y}_{\beta}^{\delta}\left(t_{0}\right)\right|^{2 q}\right),
\end{aligned}
$$

which completes the proof of (19). Finally, (20) can be straightforwardly obtained from the definition of the process $z_{\beta}^{\delta}$ and its corresponding identity in Lemma 2.

Proof of Theorem 1. Identity (19) directly implies that

$$
\mathrm{E}\left(\max _{0 \leq n \leq n_{T}}\left|\boldsymbol{y}_{t_{n}-}\right|^{2 q} \mid \mathcal{F}_{t_{0}}\right) \leq K_{2}\left(1+\left|\boldsymbol{y}_{0}\right|^{2 r}\right)
$$

which is just condition (13) of Lemma 1.

From (20), it is easy to check that the WLL approximation $\boldsymbol{y}_{\beta}^{\delta}(t-)$ is a solution to (7) for all $t \in\left[t_{n}, t_{n+1}\right]$. Thus, a straighforward application of [10, Theorem 4.5.4] to (7) yields

$$
\mathrm{E}\left(\left|\boldsymbol{y}_{t_{n+1}-}-\boldsymbol{y}_{t_{n}}\right|^{2 q} \mid \mathcal{F}_{t_{n}}\right) \leq K_{1}\left(1+\max _{0 \leq k \leq n}\left|\boldsymbol{y}_{t_{k}}\right|^{2 r}\right)\left(t_{n+1}-t_{n}\right)^{q}
$$

with $K_{1}>0$ for all $t_{n}, t_{n+1} \in(t)_{\delta}$, i.e. the condition (14) in Lemma 1 .

Conversely, since (20) holds, a direct application of [10, Lemma 5.11.7] to the Itô-Taylor expansion (17) yields

$$
\begin{aligned}
& \left|\mathrm{E}\left(\boldsymbol{F}_{\boldsymbol{p}}\left(\boldsymbol{y}_{t_{n+1}-}-\boldsymbol{y}_{t_{n}}\right)-\boldsymbol{F}_{\boldsymbol{p}}\left(\sum_{\alpha \in \Gamma_{\beta} \backslash\{\nu\}} I_{\alpha}\left[\Lambda_{\alpha}\left(t_{n}, \boldsymbol{y}_{t_{n}} ; t_{n}, \boldsymbol{y}_{t_{n}}\right)\right]_{t_{n}, t_{n+1}}\right) \mid \mathcal{F}_{t_{n}}\right)\right| \\
& \quad \leq K\left(1+\left|\boldsymbol{y}_{t_{n}}\right|^{2 r}\right)\left(t_{n+1}-t_{n}\right) \delta^{\beta}
\end{aligned}
$$

for all $t_{n}, t_{n+1} \in(t)_{\delta}$ and $\boldsymbol{p} \in P_{l}$, with $K>0$ and $r \in\{1,2, \ldots\}$. Moreover, since (18) also holds, we have

$$
\begin{aligned}
& \left|\mathrm{E}\left(\boldsymbol{F}_{\boldsymbol{p}}\left(\boldsymbol{y}_{t_{n+1}-}-\boldsymbol{y}_{t_{n}}\right)-\boldsymbol{F}_{\boldsymbol{p}}\left(\sum_{\alpha \in \Gamma_{\beta} \backslash\{v\}} I_{\alpha}\left[\lambda_{\alpha}\left(t_{n}, \boldsymbol{y}_{t_{n}}\right)\right]_{t_{n}, t_{n+1}}\right) \mid \mathcal{F}_{t_{n}}\right)\right| \\
& \quad \leq K\left(1+\left|\boldsymbol{y}_{t_{n}}\right|^{2 r}\right)\left(t_{n+1}-t_{n}\right) \delta^{\beta} \\
& \quad \leq K\left(1+\max _{0 \leq k \leq n}\left|\boldsymbol{y}_{t_{k}}\right|^{2 r}\right)\left(t_{n+1}-t_{n}\right) \delta^{\beta},
\end{aligned}
$$

which is just (15). Finally, the proof concludes by applying Lemma 1 to the WLL approximation $\boldsymbol{y}_{\beta}^{\delta}$.

Theorem 1 can also be used for obtaining the global order of weak convergence of numerical schemes derived from the WLL discretization (9). That is, by providing suitable weak approximations of desired order of convergence to the integrals (4) and (5). In this way, this result is also valuable for studying the statistical properties of inference methods that could also be derived from such WLL discretization. 


\section{Acknowledgements}

The authors are very grateful to Professor Eckhard Platen (University of Technology, Sydney) and Dr Nicola Bruti-Liberati (University of Technology, Sydney) for providing us with [1] in an early stage of preparation. Dr Nicola Bruti-Liberati was a young researcher who recently died in a traffic accident in Sydney and the authors would like to dedicate this paper to his memory. This work was partially supported by the Research Grant 03-059 RG/MATHS/LA from the Third World Academy of Science.

\section{References}

[1] Bruti-Liberati, N. (2007). Numerical solution of stochastic differential equations with jumps in finance. Doctoral Thesis, School of Finance and Economics, University of Technology, Sydney.

[2] Bruti-Liberati, N. and Platen, E. (2007). On weak predictor-corrector schemes for jump-diffusion processes in finance. To appear in Numerical Methods in Finance (Financial Math. Ser. 8), Chapman \& Hall, Boca Raton, FL.

[3] Carbonell, F., Jimenez, J. C. and Biscay, R. J. (2006). Weak local linear discretizations for stochastic differential equations: convergence and numerical schemes. J. Comput. Appl. Math. 197, 578-596.

[4] Cont, R. and Tankov, P. (2004). Financial Modelling with Jump Processes. Chapman \& Hall, Boca Raton, FL.

[5] Glasserman, P. and Merener, N. (2003). Numerical solution of jump-diffusion LIBOR market models. Finance Stoch. 7, 1-27.

[6] Higham, D. and Kloeden, P. E. (2006). Convergence and stability of implicit methods for jump-diffusion systems. Internat. J. Numer. Anal. Model. 3, 125-140.

[7] Jimenez, J. C. AND OzAKI, T. (2003). Local linearization filters for nonlinear continuous-discrete state space models with multiplicative noise. Internat. J. Control 76, 1159-1170.

[8] JimeneZ, J. C. And OZAKI, T. (2006). An approximate innovation method for the estimation of diffusion processes from discrete data. J. Time Ser. Anal. 27, 77-97.

[9] Jimenez, J. C., Biscay, R. AND OZAKI, T. (2006). Inference methods for discretely observed continuous-time stochastic volatility models: a commented overview. Asian-Pacific Financial Markets 12, 109-141.

[10] Kloeden, P. E. and Platen, E. (1995). Numerical Solution of Stochastic Differential Equations, 2nd edn. Springer, Berlin.

[11] Kubilius, K. and Platen, E. (2002). Rate of weak convergenece of the Euler approximation for diffusion processes with jumps. Monte Carlo Methods Appl. 8, 83-96.

[12] LIU, X. Q. AND LI, C. W. (2000). Weak approximations and extrapolations of stochastic differential equations with jumps. SIAM J. Numerical Anal. 37, 1747-1767.

[13] Mikulevicius, R. And Platen, E. (1998). Time discrete Taylor approximations for Itô processes with jump component. Math. Nachr. 138, 93-104.

[14] Mora, C. M. (2005). Weak exponential schemes for stochastic differential equations with additive noise. IMA J. Numerical Anal. 25, 486-506.

[15] ØKsendal, B. AND Sulem, A. (2005). Applied Stochastic Control of Jump Diffusions. Springer, Berlin.

[16] OzAKI, T. (1992). A bridge between nonlinear time series models and nonlinear stochastic dynamical systems: a local linearization approach. Statistica Sinica 2, 113-135.

[17] OzAKI, T. (1994). The local linearization filter with application to nonlinear system identification. In Proc. First US/Japan Conf. Frontiers Statist. Modeling: An Informational Approach, ed. H. Bozdogan, Kluwer, Dordrecht, pp. 217-240.

[18] Protter P. (1990). Stochastic Integration and Differential Equations. Springer, New York.

[19] Protter, P. and Talay, D. (1997). The Euler scheme for Levy driven stochastic differential equations. Ann. Prob. 25, 393-423.

[20] ShoJI, I. AND Ozaki, T. (1997). Comparative study of estimation methods for continuous time stochastic processes. J. Time Ser. Anal. 18, 485-506.

[21] ShoJI, I. AND OzAKI, T. (1998). A statistical method of estimation and simulation for systems of stochastic differential equations. Biometrika 85, 240-243.

[22] SHOJI, I. AND OzAKI, T. (1998). Estimation for nonlinear stochastic differential equations by a local linearization method. Stoch. Anal. Appl. 16, 733-752.

[23] Tuckwell, H. C. (1989). Stochastic Processes in Neurosciences. SIAM, Philadelphia, PA. 